\title{
Auxiliary diagnosis of lymph node metastasis in early gastric cancer using quantitative evaluation of sentinel node radioactivity
}

\author{
Satoshi Kamiya ${ }^{1} \cdot$ Hiroya Takeuchi $^{1} \cdot$ Tadaki Nakahara $^{2} \cdot$ Masahiro Niihara $^{1} \cdot$ \\ Rieko Nakamura $^{1}$ - Tsunehiro Takahashi ${ }^{1}$ - Norihito Wada ${ }^{1}$ - Hirofumi Kawakubo ${ }^{1}$. \\ Yoshiro Saikawa $^{1} \cdot$ Tai Omori $^{1} \cdot$ Koji Murakami $^{2} \cdot$ Yuko Kitagawa $^{1}$
}

Received: 11 September 2015/ Accepted: 6 November 2015/Published online: 30 November 2015

(c) The International Gastric Cancer Association and The Japanese Gastric Cancer Association 2015

\begin{abstract}
Background Sentinel node (SN) mapping using dye and radioisotope (RI) tracer has been reported to be feasible in cases of early gastric cancer. Because accurate diagnosis of micrometastasis is sometimes difficult in the limited time available during surgery, a faster and simpler method of improving the intraoperative diagnostic precision of lymph node metastasis is needed. The amount of tracer deposited in an SN can be determined from its radioactivity; however, the significance of the RI count has not been fully discussed. We investigated the clinical impact of the RI count when used as an adjunct to conventional lymph node dissection when diagnosing lymphatic metastasis in cases of early gastric cancer.

Methods From 2008 to 2009, patients with clinically diagnosed T1N0M0 gastric cancers who underwent gastrectomy and SN mapping were enrolled. SNs were examined by intraoperative and postoperative pathology. The RI count was measured for each SN with a handheld gamma probe; the correlation between nodal metastasis and the RI count was assessed.

Results A total of 308 SNs were harvested from 72 patients. Patients with SN metastasis had significantly higher total RI counts than those without SN metastasis ( $p=0.007)$. Among cases with $\mathrm{SN}$ metastasis, RI counts were also significantly elevated in metastasis-positive nodes, stations, and basins. In these cases, the most of SNs
\end{abstract}

Hiroya Takeuchi

htakeuchi@a6.keio.jp

1 Department of Surgery, Keio University School of Medicine, 35 Shinanomachi, Shinjuku-ku, Tokyo 160-8582, Japan

2 Department of Radiology, Keio University School of Medicine, Tokyo, Japan having the highest RI count in each case had metastasis including isolated tumor cells.

Conclusion In early gastric cancer patients, a high RI count from an SN was correlated with lymph node metastasis. Therefore, RI counting may aid efficient pathological diagnosis and focused lymph node dissection.

Keywords Gastric cancer - Sentinel lymph node biopsy · Radioactivity

\section{Introduction}

Lymph node metastasis is one of the most important prognostic factors in gastric cancer treatment. Clarifying the lymphatic network and understanding the dynamic state of lymph node metastasis are imperative for safe surgical treatment. In recent years, sentinel node (SN) navigation surgery for gastric cancer has attracted attention; this technique enables the omission of unnecessary prophylactic lymph node dissection through the efficient detection of metastatic lymph nodes [1-6]. We previously conducted a multicenter prospective trial of SN mapping for early gastric cancer using indocyanine green (ICG) solution and a radioisotope (RI)-labeled tracer, technetium- $99 \mathrm{~m}$ tin colloid solution. That study yielded favorable results from the technique, which showed high accuracy [7]. In cases without SN metastasis, minimized gastrectomy with limited lymphadenectomy can be performed $[8,9]$.

Accurate and rapid pathological examination is imperative for safe surgery based on the SN concept. However, there have been several studies showing a significant number of false-negative intraoperative lymph-node metastasis diagnoses [10]. At present, SNs are examined using frozen sections, and hematoxylin-eosin (HE) 
staining is performed for a few sections. In early gastric cancer, isolated tumor cells (ITCs) are seen, or micrometastasis (MM) involving lymph nodes is observed infrequently [11], and these are difficult to diagnose based on frozen sections and HE staining in the limited time available. Detailed pathological examination, such as multisectioning or immunohistochemical (IHC) staining, is necessary for the accurate diagnosis of these metastatic nodes. However, given the limited time available, such examinations cannot be performed on all SNs harvested during surgery.

There are few reports that detail the quantitative risk assessment of SN metastasis. Both tracer and cancer cells are delivered to lymph nodes along the lymphatic vessels. A lymph node that contains a lot of tracer may have a number of lymphatic ducts emanating from the primary lesion. In theory, there is a risk of lymph node metastasis to such an SN.

In the study reported in the present paper, we hypothesized that the amount of RI tracer in an SN reflects the risk of lymph node metastasis. Thus, higher RI counts indicate a greater risk of nodal metastasis. We assessed whether the RI count of an SN could be used to achieve a more accurate intraoperative diagnosis of metastasis during gastric cancer surgery.

\section{Materials and methods}

\section{Study subjects}

We enrolled cases of clinically diagnosed T1N0M0 adenocarcinoma of the stomach (International Union Against Cancer (UICC) TNM Classification, 6th edition, [12]) with a single lesion $<40 \mathrm{~mm}$ in size that had not been treated previously. This included endoscopic mucosal resection or endoscopic submucosal dissection, as described in our earlier study [7, 8]. In this study, we enrolled patients satisfying these indications who underwent gastrectomy with SN biopsy at Keio University Hospital (Tokyo, Japan) in 2008 and 2009. A part of this cohort has been previously reported [7]. We retrospectively investigated the characteristics of each patient and their nodal metastatic status.

\section{SN biopsy and mapping techniques}

RI tracer was administered in a similar manner to our previous study $[7,8]$. The tracer was mixed so that a final dose of $6.66 \mathrm{mCi} / 2 \mathrm{ml}$ was injected at noon on the day before surgery. Furthermore, $2.0 \mathrm{ml}$ of RI tracer were injected into the quadrants of the submucosal layer around the tumor using an endoscopic puncture needle. While under general anesthesia and before surgery, $2 \mathrm{ml}$ of $0.5 \%$ indocyanine green solution (dye tracer) were injected in the same manner [9]. We diagnosed lymph nodes that were positive for either indocyanine green (ICG) staining or RI tracer as SNs. Because reliable tracer administration is crucial to accurate SN mapping, we used a few well-seasoned operators for tracer injection to minimize handling bias. SNs were harvested and the RI count of each SN was measured for $10 \mathrm{~s}$ with a handheld gamma probe at the right angle. For instant diagnosis, harvested SNs were examined during surgery via frozen sections and $\mathrm{HE}$ staining in a maximum sectioned surface. After surgery, these specimens were embedded in paraffin and additionally examined by HE staining and IHC examination with cytokeratin staining.

We investigated the diagnostic accuracy of intraoperative examinations of $\mathrm{SNs}$ as a representation of metastatic status and also assessed the correlation of $\mathrm{SN}$ metastasis with the RI count of SNs, lymphatic stations, and basins in each case. Lymphatic basins were defined as lymphatic areas divided according to the following 5 directions along the main arteries, as previously described [13]: the left gastric artery area (1-GA) consisted of lymph node stations 1 and 7 and the left two-thirds of station 3; the right gastric artery area (r-GA) consisted of stations 5 and $8 \mathrm{a}$ and the right one-third of station 3; the right gastroepiploic artery area (r-GEA) consisted of stations $4 \mathrm{~d}$ and 6 ; the left gastroepiploic artery area (1-GEA) consisted of stations 4sa and $4 \mathrm{sb}$; and the posterior gastric artery (p-GA) consisted of station $11 \mathrm{p}$.

\section{Surgical procedure}

In this study, we performed distal gastrectomy, proximal gastrectomy, and pylorus-preserving gastrectomy with D1+ or D2 lymph node dissection as described in the Gastric Cancer Treatment Guideline [14]. We performed conventional standard gastrectomy with D2 lymph node dissection in patients with metastatic SNs.

\section{Relevance of the RI count and nodal metastasis}

To evaluate the relevance of the RI count to nodal metastasis, we investigated the nodal RI count, the total nodal RI count, and the number of SNs detected in each case. In cases with $\mathrm{SN}$ metastasis, we also investigated the RI counts of the nodes, stations, and basins and calculated 
the ratios of those RI counts with the total nodal RI count, as defined below. These RI count ratios indicate the relative amounts of tracer in SNs, stations, and basins in each case. Furthermore, we also ordered the nodes according to RI count and then investigated the correlation of this order of nodes with nodal metastasis.

$$
\begin{aligned}
\text { Nodal RI count ratio }(\%)= & \frac{\text { RI counts of the } \mathrm{SNs}}{\text { Total nodal RI count for this case }} \\
& \times 100
\end{aligned}
$$

Basin RI count ratio $(\%)=\frac{\text { Sum of RI counts of the basins }}{\text { Total nodal RI count for this case }}$ $\times 100$

\section{Informed consent}

The method of identifying SNs by dye and RI was reviewed and approved by the Institutional Review Board of the Keio University School of Medicine. Before surgery, informed consent was obtained from all subjects.

\section{Statistical analysis}

All statistical analyses were performed with EZR (Saitama Medical Center, Jichi Medical University, Saitama, Japan), a graphical user interface for $\mathrm{R}$ (The $\mathrm{R}$ Foundation for Statistical Computing, Vienna, Austria); more precisely, it is a modified version of the $\mathrm{R}$ commander with added statistical functions that are frequently used in biostatistics. Fisher's exact and chi-square tests were performed to compare differences in the characteristics of the enrolled patients. To compare the means and medians, we performed Student's $t$-test assuming unequal variances, in addition to the Mann-Whitney test. Differences were considered significant if the $p$ value from a two-sided test was $<0.05$.

\section{Results}

\section{Patient characteristics}

A total of 308 SNs were harvested from 72 patients, and 18 nodes were confirmed as metastatic in 11 patients. Patient characteristics are shown in Table 1 . The medians of the

\begin{tabular}{|c|c|c|}
\hline Parameter & & Value \\
\hline Age & & $61.5 \pm 12.7$ \\
\hline Sex & male/female & $43(60 \%) / 29(40 \%)$ \\
\hline Invasion depth & $\mathrm{M} / \mathrm{SM}$ & $28(39 \%) / 44(61 \%)$ \\
\hline Longest diameter & $(\mathrm{mm})$ & $25.2 \pm 9.71$ \\
\hline \multicolumn{3}{|l|}{ Long axis } \\
\hline Upper third & & $11(15 \%)$ \\
\hline Middle third & & $42(58 \%)$ \\
\hline Lower third & & $19(26 \%)$ \\
\hline \multicolumn{3}{|l|}{ Circumference } \\
\hline Lesser curvature & & $31(43 \%)$ \\
\hline Greater curvature & & $12(17 \%)$ \\
\hline Anterior wall & & $10(14 \%)$ \\
\hline Posterior wall & & $19(26 \%)$ \\
\hline \multicolumn{3}{|l|}{ Tumor differentiation } \\
\hline Well & & $17(24 \%)$ \\
\hline Moderate & & $12(17 \%)$ \\
\hline Poor & & $43(60 \%)$ \\
\hline \multicolumn{3}{|l|}{ Vascular invasion } \\
\hline Negative & & $64(89 \%)$ \\
\hline Positive & & $8(11 \%)$ \\
\hline \multicolumn{3}{|l|}{ Lymphatic invasion } \\
\hline Negative & & $52(72 \%)$ \\
\hline Positive & & $20(28 \%)$ \\
\hline Number of SNs detected & Median (range) & $4(1-10)$ \\
\hline Total nodal RI count & Median (range) & $774.5(0-18021)$ \\
\hline
\end{tabular}
number of SNs detected and the total nodal RI count were 4 and 774.5 , respectively.
Table 1 Patient characteristics ( $n=72$ patients)

$M$ mucosal, $R I$ radioisotope, $S M$ submucosal, $S N$ sentinel node

\section{Diagnosis of SNs and pathological examination}

In $65(90 \%)$ of the 72 subjects, SNs were intraoperatively diagnosed as being metastasis negative; seven patients were $\mathrm{SN}$ metastasis positive by intraoperative pathological examination (Fig. 1). Non-SN metastases were diagnosed by postoperative pathological examination in three cases which had SN metastases. Isolated tumor cells (ITCs) were diagnosed in five SNs from four cases, and those ITCs were initially diagnosed by postoperative detailed pathological examination using IHC. Those cases did not have metastases in the non-SNs. Therefore, in this study, there were no false-negative cases as determined by $\mathrm{SN}$ analysis. However, when the ITCs were considered to be metastases, the diagnostic precision of intraoperative diagnosis using frozen sections and HE staining was not high. Indeed, the sensitivity, negative predictive value (NPV), and accuracy were 63.6 (7/11), 93.8 (61/65), and $94.4 \%$ (68/72), respectively.

We divided the cases into the following two groups: those that had SN metastasis and those that did not. There was a greater number of detected SNs $(p=0.001)$ and a 


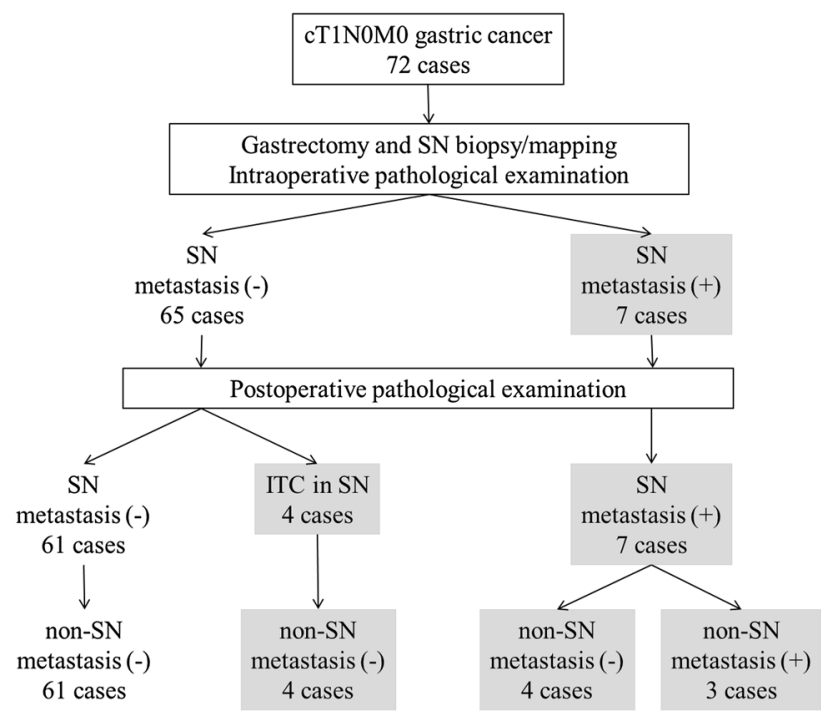

Fig. 1 Nodal metastasis status and the precision of intraoperative and postoperative pathological diagnoses. Nodal metastases were diagnosed in seven cases by intraoperative examination, while four cases that were not diagnosed intraoperatively showed ITCs in detailed postoperative examination. Only three non-SN metastases were found, and these three were identified in cases with SN metastases

significantly higher RI count $(p=0.007)$ in the SN metastasis-positive group (Table 2).

The median total nodal RI count was estimated to be 774.5. In the patients with RI counts that were higher than the median, the nodal metastasis rate was $25.0 \%(9 / 36)$, whereas the nodal metastasis rate was only $5.6 \%(2 / 34)$ in the patients with RI counts that were lower than the median. The difference between these two groups was significant $(p=0.04)$. The mean RI count in the $18 \mathrm{SNs}$ that presented metastasis was 960.7 (range 27-7805), which was significantly higher than the mean RI count in the 290 SNs that did not show metastasis (479.5; range 0-8316) $(p=0.01)$.

\section{Correlation of the RI count with nodal metastasis among cases with $\mathrm{SN}$ metastasis}

To identify whether quantitatively measuring the RI count improved intraoperative diagnostic precision, we investigated the correlation of the RI count and RI count ratio with nodal metastasis (Table 3). We assessed $56 \mathrm{SNs}$ from 11 cases with SN metastasis (18 SNs were metastatic in total). The RI count ratios for metastatic SNs were significantly higher than those for non-metastatic SNs ( $p=0.037$ ). Furthermore, the RI count ratios of the lymphatic stations and basins with metastatic SNs were also significantly higher than those without metastatic SNs.

The RI count order and the presence of metastasis in the SNs are shown for each case with a metastatic SN in Table 4. The SNs with metastasis had higher RI counts. We arranged these 56 SNs from the 11 cases that had SN metastasis in order of descending RI count for each case. In 8 cases $(72.7 \%)$ the $\mathrm{SN}$ with the highest RI count had metastasis, and in 9 cases $(81.8 \%)$ the SNs with the two highest RI counts had metastasis; 10 cases (90.9\%) had metastasis in the SNs with the three highest RI counts. Using detailed pathology to examine only the SN with the highest count, we were able to diagnose metastasis with a greater precision than the conventional detection method. Thus, sensitivity, NPV, and accuracy were 72.7 (8/11), 95.3 (61/64), and 95.8\% (69/72), respectively. Similarly, a more accurate diagnosis was conducted by checking the three SNs with the highest RI counts (Table 5). RI counting and ranking nodes based on the RI count can help to diagnose the nodal metastases more precisely than the conventional method of using $\mathrm{HE}$ staining and frozen sections. We found nodal metastasis in the fifth of five SNs in just one case (case 7 in Table 5); however, this metastatic node showed macrometastasis and could be diagnosed by conventional intraoperative examination.
Table 2 Comparison of the number of SNs detected and the total RI count between the cases with and without SN metastasis (total number of cases $n=72$ )

Table 3 Correlation between metastasis and the RI count ratios of SNs, stations, and basins, based on 11 cases with nodal metastasis

\begin{tabular}{lllll}
\hline & & $\begin{array}{l}\text { SN metastasis }(-) \\
n=61\end{array}$ & $\begin{array}{l}\text { SN metastasis }(+) \\
n=11\end{array}$ & $p$ value \\
\hline Number of SNs detected & Median (range) & $3(1-10)$ & $5(2-9)$ & 0.001 \\
Total nodal RI count & Median (range) & $612(0-10679)$ & $1983(523-18021)$ & 0.007 \\
\hline
\end{tabular}

$R I$ radioisotope, $S N$ sentinel node

\begin{tabular}{lllll}
\hline & & \multicolumn{2}{l}{ RI count ratio $(\%)$} & \multirow{2}{*}{$p$ value } \\
\cline { 2 - 4 } & & Metastasis $(-)$ & Metastasis $(+)$ & \\
\hline Node $(n=56)$ & Median (range) $n$ & $9.8(1.2-57.6) 38$ & $22.5(0.9-73.4) 18$ & 0.037 \\
Station $(n=25)$ & Median (range) $n$ & $22.6(1.2-66.7) 12$ & $70.7(3.6-100) 13$ & 0.001 \\
Basin $(n=20)$ & Median (range) $n$ & $22.8(1.2-74.1) 9$ & $91.6(3.6-100) 11$ & 0.003 \\
\hline
\end{tabular}

$R I$ radioisotope 
Table 4 SNs arranged in order of descending RI count

\begin{tabular}{|c|c|c|c|c|c|c|c|c|c|}
\hline \multirow[t]{2}{*}{ Case } & \multicolumn{8}{|l|}{ RI count } & \multirow[t]{2}{*}{ Total nodal RI count } \\
\hline & $\mathrm{SN}-1$ & $\mathrm{SN}-2$ & $\mathrm{SN}-3$ & $\mathrm{SN}-4$ & SN-5 & SN-6 & $\mathrm{SN}-7$ & $\mathrm{SN}-8$ & \\
\hline 1 & 384 & 139 & & & & & & & 523 \\
\hline 2 & $348(I T C)$ & 150 & 58 & $46(I T C)$ & & & & & 602 \\
\hline 3 & 330 & 225 & 203 & 27 & & & & & 785 \\
\hline 4 & 512 & 343 & 169 & 22 & & & & & 1046 \\
\hline 5 & $934(I T C)$ & 452 & 378 & 219 & & & & & 1983 \\
\hline 6 & 1534 & 718 & 152 & 150 & 148 & & & & 2702 \\
\hline 7 & 2546 & 2483 & 1682 & 571 & 271 & & & & 7553 \\
\hline 8 & 7805 (ITC) & 6000 & 2053 & 1298 & 865 & & & & 18021 \\
\hline 9 & 1308 & 317 & $315(I T C)$ & 287 & 281 & 194 & 148 & & 2850 \\
\hline 10 & 689 & 316 & 236 & 145 & 144 & 96 & 57 & 27 & 1710 \\
\hline 11 & 8316 & 2532 & 874 & 854 & 636 & 600 & 440 & 178 & 14430 \\
\hline
\end{tabular}

Bold italics indicate metastatic SNs

ITC isolated tumor cell, $R I$ radioisotope, $S N$ sentinel node

Table 5 Comparison of the diagnostic abilities of the conventional method and the RI count method of diagnosing lymph node metastasis

\begin{tabular}{lllll}
\hline \multicolumn{5}{c}{ Method used for the diagnosis of nodal metastasis } \\
\cline { 2 - 5 } & Conventional & SN with the highest RI count & Two SNs with the highest RI counts & Three SNs with the highest RI counts \\
\hline Sensitivity & $63.6 \%(7 / 11)$ & $72.7 \%(8 / 11)$ & $81.8 \%(9 / 11)$ & $90.9 \%(10 / 11)$ \\
NPV & $93.8 \%(61 / 65)$ & $95.3 \%(61 / 64)$ & $96.8 \%(61 / 63)$ & $98.4 \%(61 / 62)$ \\
Accuracy & $93.1 \%(68 / 72)$ & $95.8 \%(69 / 72)$ & $97.2 \%(70 / 72)$ & $98.6 \%(71 / 72)$ \\
\hline
\end{tabular}

Conventional method involved using frozen sections and HE staining for all detected SNs

$N P V$ negative predictive value, $R I$ radioisotope, $S N$ sentinel node

Incidentally, this case had a high total RI count of 7553 , which was much larger than the median value.

\section{Discussion}

For SN mapping, we administered a dye and RI tracer, and this tracer was delivered to the lymph nodes via lymphatic ducts. The dynamic state of the tracer is thought to reflect the spread of cancer cells; thus, the distribution of tracer through the SNs is assumed to resemble the network of lymphatic flow. We hypothesized that the RI count of a node indicates the amount of tracer deposited in that node, and that high RI counts demonstrate greater lymphatic drainage from the primary lesion and a higher incidence of nodal metastasis. In this work, we investigated whether the RI count or ratio could be used to predict the status of lymph node metastasis in early gastric cancer cases.

In this study, 7 of 72 cases had SN metastases that were diagnosed during surgery, and 5 SNs from 4 cases were additionally found to harbor ITCs. All of the cases that had metastatic non-SN lymph nodes also had metastatic SNs. These results agree with data from other studies assessing the SN concept of other carcinomas [1-3, 1517].

Regarding nodal metastasis and RI count, the number of SNs detected and the measured RI counts in metastatic cases were significantly higher than those in nonmetastatic cases. This finding suggests that nodal metastasis is associated with a high RI count in SNs.

We calculated the RI count ratios for SNs, stations, and basins. These RI count ratios showed the distribution of the administered tracer in each case. Our results showed that the RI count ratios of lymph nodes, lymphatic stations, and basins with metastases were significantly higher than those of nonmetastatic SNs, stations, and basins. High concentrations of tracer were observed in SNs, stations, and basins that were metastatic.

This study demonstrated that an uneven distribution of tracer may be correlated with nodal metastasis. When we arranged the SNs in order of RI count, most metastases were found in the three nodes with the highest RI counts. We propose that nodal metastases can be diagnosed with greater precision by performing a detailed examination of the three nodes with the highest RI counts in each patient. The RI count can even highlight ITCs that are not found in 
conventional intraoperative examinations which use frozen sections and HE staining of all detected SNs. Moreover, it is possible that examining the $\mathrm{SN}$ with the highest RI count could allow the metastatic status to be gauged more precisely than achieved using the conventional method. However, not all metastatic nodes have a high RI count, so we instead propose that the RI count can compensate for the limitations of existing pathological diagnosis. A more accurate diagnosis could be obtained by performing sequential RI counting.

Regarding micrometastasis: although the significance of an ITC is unclear, it cannot be ignored, because lymph node metastasis is an important prognostic factor for gastric cancer [18, 19]. Pathological examination by multiple sections, IHC staining, or RT-PCR is required to detect ITCs. However, it is difficult to perform these examinations in the limited surgical period available [20, 21]. A simpler and easier method of improving intraoperative diagnostic precision is needed for the accurate and fast diagnosis of SN metastasis, including ITCs. Recently, Kumagai et al. reported that one-step nucleic acid amplification (OSNA) assay presents a diagnostic ability equivalent to that of the postoperative 2-mm-interval histological examination. This assay should prove a useful tool for the intraoperative diagnosis of lymph node micrometastasis in gastric cancer surgery [22].

In cases with a high RI count, station or basin dissection should be performed to check for nodal metastasis even when metastasis was not found in a rapid pathological examination. In contrast, low RI counts in SNs indicate a lower probability of metastasis. Combining sequential RI counting with a close examination, such as 2-mm-interval section or OSNA, will lead to a more accurate diagnosis and greater patient safety through the minimization of lymphadenectomy based on SN status.

Although this study showed that RI counts and count ratios were relatively high in cases with metastasis, the reason for this is yet to be clarified. To some extent, it may be due to an uneven distribution of the tracer: with or without metastasis, each case may have areas that differ in the amount of incoming tracer. Alternatively, the anatomic microstructure may be inadvertently modified by metastasis, which would change the amount of incoming tracer. To date, although there have been no reports showing that metastasis directly increases the number of SNs and the RI count, some reports do provide a clue regarding this issue. Several studies have shown that vascular endothelial growth factor (VEGF)-C or VEGF-D produced by tumor cells enables the induction of lymphangiogenesis but it also enhances lymphatic metastasis to SNs [23-25]. Lymphangiogenesis in regional lymph nodes plays an early role in spreading tumor cells though the lymphatic system to distant organs in gastric cancer. Nagata et al. observed morphological changes in lymph nodes during the establishment of lymph node metastasis using a rat mesenteric SN model [26]. Watanabe et al. reported that intranodal lymphatic vessel density was significantly correlated with the size of the metastasis [27]. Furthermore, peritumoral lymphovascular density was significantly increased in cases with lymph node metastasis [28]. These results suggest that the lymphatic network develops both morphologically and immunologically in cases with lymph node metastasis. This development of the lymphatic network may affect the way in which the tracer drifts from the primary lesion and may lead to the high number of SNs and high RI counts seen in metastatic cases. To confirm this mechanism, a comparison between the dynamic status of the tracer particles and that of the metastatic cancer cells must be performed, along with other basic studies of, for example, the epithelial-mesenchymal transition [29]. In this study, we showed that RI tracer is a useful tool, as it allows us to visualize the lymphatic network and to quantify the risk of nodal metastasis.

Limitations of this study include measurement error due to the precision of tracer administration and a change in the RI count caused by the decrease in radiation over time. It was technically difficult to administer the same quantity by endoscopic injection in every case. Furthermore, the radiation half-life of technetium-99m tin colloid is $6 \mathrm{~h}$, so we cannot exclude the possibility that there is some measurement error caused by the fact that the radiation emitted depends on the time that RI counting was performed. To minimize these errors, only a select few operators were allowed to administer the tracer and to perform the surgery, which was always carried out at the same fixed time after tracer administration, and we compared RI counts using RI count ratios. The radioactivity of the RI tracer that was administered to the same patient would be attenuated equally, and RI counts were measured at the same time during the operation. Therefore, in comparison of the RI count ratios for different cases, it is not necessary to adjust those values for errors resulting from the decrease in radiation over time. Using this comparison method, we can minimize the impact of measurement errors on the results of this study. The influence of these errors should be inspected in a future prospective study. Furthermore, given that they rely on only 11 patients who were $\mathrm{SN}$ metastasis positive, the results of this study should be accepted with considerable caution. Yaguchi et al. suggested that RI counting is a feasible approach for identifying metastatic SNs [30], and the results of the present study are similar to their results, which would support the novel findings of that group.

When quantitatively evaluating the radioactivity of the RI tracer, we must account for the decrease in radioisotope 
activity over time. In our study, we employed the RI count ratio to minimize this problem. In addition, we were able to directly compare the relative amount of RI tracer present in a metastatic case with the relative amount of RI tracer present in a nonmetastatic case using the RI count ratio. We believe that the RI count ratio allows the risk of SN metastasis to be easily and simply estimated without the need to adjust for the decrease in radioisotope activity over time.

In summary, we have demonstrated that there is a close relationship between nodal metastasis and the RI count of an SN. In addition to conventional rapid pathology, quantitative RI counting could permit a more accurate intraoperative diagnosis of lymph node metastasis, thus enabling safe and minimized surgery for early gastric cancer.

\section{Compliance with ethical standards}

Conflicts of interest Yuko Kitagawa has received grants from Daiichi Sankyo Co., Ltd., Covidien; Taiho Pharmaceutical Co., Ltd., Olympus Corporation; and Nihon Medi-Physics Co., Ltd.

Ethical standards All procedures were conducted in accordance with the ethical standards of the responsible committee on human experimentation (institutional and national) and with the Helsinki Declaration of 1964 and later versions. Informed consent or a substitute for it was obtained from all patients included in the study.

\section{References}

1. Miwa K, Kinami S, Taniguchi K, Fushida S, Fujimura T, Nomura A. Mapping sentinel nodes in patients with early-stage gastric carcinoma. Br J Surg. 2003;90:178-82.

2. Kitagawa Y, Fujii H, Mukai M, Kuboda T, Ando N, Watanabe M, et al. The role of the sentinel lymph node in gastrointestinal cancer. Surg Clin North Am. 2000;80(6):1799-809.

3. Kitagawa Y, Kitajima M. Gastrointestinal cancer and sentinel node navigation surgery. J Surg Oncol. 2002;79(3):188-93.

4. Mayanagi S, Takeuchi H, Kamiya S, Niihara M, Nakamura R, Takahashi $T$, et al. Suitability of sentinel node mapping as an index of metastasis in early gastric cancer following endoscopic resection. Ann Surg Oncol. 2014;21(9):2987-93.

5. Takeuchi H, Oyama T, Kamiya S, Nakamura R, Takahashi T, Wada N, et al. Laparoscopy-assisted proximal gastrectomy with sentinel node mapping for early gastric cancer. World J Surg. 2011;35(11):2463-71.

6. Kitagawa Y, Fujii H, Mukai M, Kubota T, Otani Y, Kitajima M. Radio-guided sentinel node detection for gastric cancer. $\mathrm{Br} \mathrm{J}$ Surg. 2002;89(5):604-8.

7. Kitagawa Y, Takeuchi H, Takagi Y, Natsugoe S, Terashima M, Murakami N, et al. Sentinel node mapping for gastric cancer: a prospective multicenter trial in Japan. J Clin Oncol. 2013;31(29):3704-10.

8. Takeuchi H, Kitagawa Y. New sentinel node mapping technologies for early gastric cancer. Ann Surg Oncol. 2013;20:522-32.

9. Tajima Y, Murakami M, Yamazaki K, Masuda Y, Kato M, Sato A, et al. Sentinel node mapping guided by indocyanine green fluorescence imaging during laparoscopic surgery in gastric cancer. Ann Surg Oncol. 2010;17(7):1787-93.

10. Miyashiro I, Hiratsuka M, Sasako M, Sano T, Mizusawa J, Nakamura K, et al. High false-negative proportion of intraoperative histological examination as a serious problem for clinical application of sentinel node biopsy for early gastric cancer: final results of the Japan Clinical Oncology Group Multicenter Trial JCOG0302. Gastric Cancer. 2014;17(2):316-23.

11. Yanagita S, Natsugoe S, Uenosono Y, Arigami T, Arima H, Kozono $\mathrm{T}$, et al. Detection of micrometastases in sentinel node navigation surgery for gastric cancer. Surg Oncol. 2008;7(3):203-10.

12. International Union Against Cancer. TNM classification of malignant tumours. 7th ed. Chichester: Wiley; 2009.

13. Kinami S, Fujimura T, Ojima E, Fushida S, Ojima T, Funaki H, et al. PTD classification: proposal a new classification of gastric cancer location based on physiological lymphatic flow. J Clin Oncol. 2008;13:320-9.

14. Japanese Gastric Cancer Association. Japanese gastric cancer guidelines 2010 (ver. 3). Gastric Cancer. 2011;14:113-23.

15. Morton DL, Wen DR, Wong JH, Economou JS, Cagle LA, Storm FK, et al. Technical details of intraoperative lymphatic mapping for early stage melanoma. Arch Surg. 1992;127(4):392-9.

16. Krag SN, Weaver DL, Alex JC, Fairbank JT. Surgical resection and radiolocalization of the sentinel lymph node in breast cancer using a gamma probe. J Surg Oncol. 1993;2(6):335-9 discussion 340.

17. Giuliano AE, Kirgan DM, Guenther JM, Morton DL. Lymphatic mapping and sentinel lymphadenectomy for breast cancer. Ann Surg. 1994;220(3):391-8 discussion 398-401.

18. Fukagawa T, Sasako M, Ito S, Nakanishi H, Inuma H, Natsugoe $\mathrm{S}$, et al. The prognostic significance of isolated tumor cells in the lymph nodes of gastric cancer patients. Gastric Cancer. 2010;13:191-6.

19. Horstmann O, Füzesi L, Markus PM, Werner C, Becker H. Significance of isolated tumor cells in lymph nodes among gastric cancer patients. J Cancer Res Clin Oncol. 2004;130(12):733-40.

20. Morita D, Tsuda H, Ichikura T, Kimura M, Aida S, Kosuda S, et al. Analysis of sentinel node involvement in gastric cancer. Clin Gastroenterol Hepatol. 2007;5(9):1046-52.

21. Shimizu Y, Takeuchi H, Sakakura Y, Saikawa Y, Nakahara T, Mukai M, et al. Molecular detection of sentinel node micrometastases in patients with clinical NO gastric carcinoma with real-time multiplex reverse transcription-polymerase chain reaction assay. Ann Surg Oncol. 2012;19(2):469-77.

22. Kumagai K, Yamamoto N, Miyashiro I, Tomita Y, Katai H, Kushima R, et al. Multicenter study evaluating the clinical performance of the OSNA assay for the molecular detection of lymph node metastases in gastric cancer patients. Gastric Cancer. 2014;17(2):273-80.

23. Mandriota SJ, Jussila L, Jeltsch M, et al. Vascular endothelial growth factor-C-mediated lymphangiogenesis promotes tumour metastasis. EMBO J. 2001;20:672-82.

24. Karpanen T, Egeblad M, Karkkainen MJ, et al. Vascular endothelial growth factor C promote tumor lymphangiogenesis and intralymphatic tumor growth. Cancer Res. 2001;61:1786-90.

25. Takeuchi H, Kitagaway Y. Sentinel node and mechanism of lymphatic metastasis. Ann Vasc Dis. 2012;5(3):249-57.

26. Nagata H, Arai T, Soejima Y, Suzuki H, Ishii H, Hibi T. Limited capability of regional lymph nodes to eradicate metastatic cancer cells. Cancer Res. 2004;64:8239-48.

27. Watanabe M, Tanaka H, Ohira M, Yoshii M, Sakurai K, Toyokawa $\mathrm{T}$, et al. Intranodal lymphangiogenesis precedes development of lymph node metastasis and accelerates progression of gastric cancer. J Gastrointest Surg. 2014;18:481-90. 
28. Pak KH, Jo A, Choi HJ, et al. The different role of intramural and peritumoral lymphangiogenesis in gastric cancer progression and prognosis. BMC Cancer. 2015;15:498.

29. Greenburg G, Hay ED. Epithelia suspended in collagen gels can lose polarity and express characteristics of migrating mesenchymal cells. J Cell Biol. 1982;95(1):333-9.
30. Yaguchi Y, Tsujimoto H, Hiraki S, et al. Preferentially examined sentinel nodes for sentinel node navigation surgery in gastric cancer. Mol Clin Oncol. 2015;3:944-8. 